\title{
Efficacy of Magnesium Sulfate on Maternal Mortality in Eclampsia
}

Jaskamal Padda ${ }^{1}$, Khizer Khalid ${ }^{1}$, Lanson B. Colaco ${ }^{1}$, Sandeep Padda ${ }^{1}$, Nymisha L. Boddeti ${ }^{1}$, Armughan S. Khan ${ }^{1}$, Ayden Charlene Cooper ${ }^{1}$, Gutteridge Jean-Charles ${ }^{2,1}$

1. Internal Medicine, JC Medical Center, Orlando, USA 2. Internal Medicine, Advent Health \& Orlando Health Hospital, Orlando, USA

Corresponding author: Nymisha L. Boddeti, nymisha.boddeti@gmail.com

\begin{abstract}
Eclampsia is a common complication of preeclampsia patients and can be life-threatening for both the mother and the fetus. Hence, timely intervention and appropriate management of this detrimental condition are extremely crucial. Eclampsia is described as the occurrence of generalized convulsions in patients with preeclampsia. Magnesium sulfate $\left(\mathrm{MgSO}_{4}\right)$ is the drug of choice for treating and preventing eclampsia. This review aims to study and analyze the available literature on the pathogenesis of eclampsia, the pharmacology of $\mathrm{MgSO}_{4}$, and its effectiveness in the management of eclampsia. Other proposed treatments and their comparative study with $\mathrm{MgSO}_{4}$ are also discussed. Additionally, we examine the data regarding the impact of eclampsia, its public health burden, and the cost-effectiveness of $\mathrm{MgSO}_{4}$. One of the major drawbacks associated with the use of $\mathrm{MgSO}_{4}$ in low-income countries has been the cost of treatment and the lack of resources. We have analyzed the trials that have proposed alternate treatment regimens which could shape new guidelines to resolve these issues. For this review, we extensively studied abstract and full-text articles from multiple databases. This article discusses the pathophysiology of eclampsia, the pharmacology of $\mathrm{MgSO}_{4}$, the issues surrounding eclampsia management, and how $\mathrm{MgSO}_{4}$ benefits these patients.
\end{abstract}

Categories: Emergency Medicine, Internal Medicine, Obstetrics/Gynecology

Keywords: magnesium sulfate, mgso4, eclampsia, preeclampsia, cost-effectiveness, maternal mortality, obstetric medicine, risk of seizure

\section{Introduction And Background}

Eclampsia is a known deleterious sequela of preeclampsia. It has been defined as new-onset generalized tonic-clonic seizures in patients with preeclampsia. Preeclampsia is the occurrence of hypertension (HTN) after 20 weeks of gestation with concurrent proteinuria/end-organ dysfunction [1]. Annually, both eclampsia and preeclampsia account for nearly 63,000 maternal deaths worldwide [2]. A study conducted by the Centers for Disease Control and Prevention (CDC) found an overall case-fatality rate of preeclampsia and eclampsia to be 6.4 per 10,000 cases at delivery. This study also found an increased risk of death in patients at 20-28 weeks of gestation and 3.1 times increased incidence of preeclampsia/eclampsia in black women compared to white women [3].

Eclamptic seizures are a medical emergency and can arise after 20 weeks of gestation, either antepartum, intrapartum, or postpartum. They require urgent intervention to prevent death in the mother and fetus [1]. Magnesium sulfate $\left(\mathrm{MgSO}_{4}\right)$ has been proven to be an effective first-line treatment for the prevention and treatment of eclampsia $[1,4]$. It should be continued for at least 24 hours from the time of the last seizure and the patient should be monitored closely for toxicity [1]. Even with the risk of side effects and toxicity, many studies have proven that $\mathrm{MgSO}_{4}$ is much more superior in the treatment of eclampsia compared to other drugs $[4,5]$. Nevertheless, the prevalence of eclampsia has decreased because of enhanced prenatal care, judicious use of medical therapy (blood pressure control, seizure prophylaxis, etc.), and conducting term deliveries by either induction or cesarean section [6]. In this review, we discuss the mechanism of eclampsia, the pharmacokinetics and pharmacodynamics of $\mathrm{MgSO}_{4}$, the efficacy of $\mathrm{MgSO}_{4}$ in reducing mortality in eclamptic patients, and the challenges encountered in $\mathrm{MgSO}_{4}$ administration.

\section{Review}

\section{Pathophysiology of eclampsia}

Although a comprehensive review of the pathophysiology is beyond the scope of this article, a brief mention of some of the key elements involved in the pathophysiology of eclampsia has been discussed. Eclampsia is a complication of preeclampsia, which is why it is important to understand the pathogenesis of preeclampsia. The placenta is considered to play a significant role in the pathophysiology of preeclampsia. After the placenta is delivered, the symptoms subside quickly [7]. The primary issue is that placental-derived 
factors released into the maternal circulation cause diffuse endothelial dysfunction, vasoconstriction, and increased vascular permeability, resulting in HTN, proteinuria, and edema [8].

The main pathophysiological occurrences can be understood in a simplified manner using the widely accepted "two-stage theory," which states that preeclampsia develops in two stages. The first is decreased placental perfusion (Stage 1), which results in the release of factors that cause generalized systemic pathology (Stage 2) [9]. The rest of the pathophysiology is explained in Figure 1 [8-11].

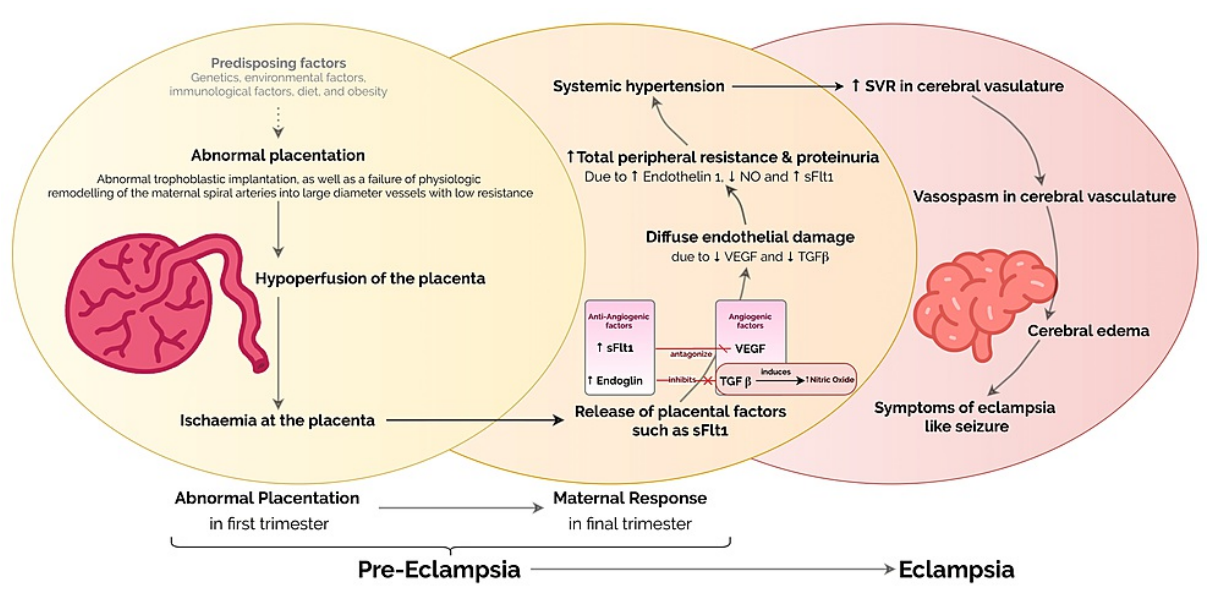

FIGURE 1: Pathophysiology of preeclampsia and eclampsia.

VEGF: vascular endothelial growth factor; NO: nitric oxide; sFItl: soluble FMS-like tyrosine kinase; TGF $\beta$ : transforming growth factor-beta; SVR: systemic vascular resistance

The image was created by the author (Lanson B. Colaco, MBBS).

According to studies, eclampsia is caused by both cerebral edema and vasospasm. Edema can be generalized or localized, such as in the occipital lobes, white matter, or watershed regions. Vasospasm has been observed in both angiographic and Doppler studies leading to ischemia, causing reduced perfusion of the brain. All of this culminates in edema and microinfarcts (and, on rare occasions, hemorrhage) in these patients [12-15]. Because cerebral edema develops within the restricted area of the skull, it causes progressive brain compression as well as the usual neurologic symptoms of eclampsia such as headache, nausea, vomiting, cortical blindness, and convulsions $[16,17]$.

\section{Pharmacokinetics and pharmacodynamics of magnesium sulfate}

Regimens

$\mathrm{MgSO}_{4}$ is usually administered by either intramuscular (IM) or intravenous (IV) routes. The two standard regimens that are most commonly used for the management of severe preeclampsia and eclampsia are predominantly the IM Pritchard regimen and the exclusively IV Zuspan regimen [18]. The Pritchard regimen involves administering two loading doses of $\mathrm{MgSO}_{4}$, consisting of a slow IV dose of $4 \mathrm{~g}$ over five to ten minutes, immediately followed by an IM dose of $10 \mathrm{~g}$ divided into $5 \mathrm{~g}$ in each buttock. This is then followed by maintenance dosing with $5 \mathrm{~g}$ IM into the alternate buttocks every four hours [19]. In the Zuspan regimen, a single loading dose of $4 \mathrm{~g}$ is administered as a slow IV infusion over five to ten minutes, followed by an hourly maintenance infusion of 1-2 $\mathrm{g}$ by a controlled infusion pump [20]. The serum concentrations of magnesium obtained from the Pritchard regimen fluctuate more often compared to the Zuspan regimen [21].

Pharmacokinetics

After administration, about $40 \%$ of plasma magnesium becomes protein-bound, while the unbound ionized magnesium increases proportionately to the total serum concentration of magnesium [22]. The free magnesium ions diffuse into the extravascular space, into the bone, and across the placenta and fetal membranes to enter the fetus and amniotic fluid [23-25]. Magnesium is eliminated almost exclusively by the maternal kidneys, with almost $90 \%$ of the dose excreted in the urine during the first 24 hours after an IV infusion [26]. A plasma concentration of 1.8-3.0 mmol/L is recommended for the treatment of eclamptic seizures while carefully monitoring for toxicity beyond this recommended concentration. The first warning of imminent toxicity in the mother is the loss of the patellar reflex at concentrations between 3.5 and 5 $\mathrm{mmol} / \mathrm{L}[26]$. Respiratory paralysis and cardiac arrest may occur at supratherapeutic concentrations beyond 5 $\mathrm{mmol} / \mathrm{L}$ [27]. Therefore, close monitoring for the loss of deep tendon reflexes, respiratory rate $<12$ 
breaths/minute, urine output $<30 \mathrm{~mL} /$ hour, and high plasma concentrations are of paramount importance [28].

Pharmacodynamics

The anticonvulsant mechanism of $\mathrm{MgSO}_{4}$ is attributed to its actions upon the central nervous system (CNS) and vascular endothelium, while its effects are mediated through the neuromuscular junction (NMJ). Generalized CNS depression occurs via voltage-dependent N-methyl-D-aspartate (NMDA) receptor blockade [29] and NMJ blockade by decreasing calcium conductance, acetylcholine release, and motor endplate excitability to acetylcholine release [30]. It is suggested to cause vasodilation by stimulating prostacyclin I2 and nitric oxide synthesis in vascular endothelial cells [31]. In addition, it is understood that this vasodilatory effect of $\mathrm{MgSO}_{4}$ upon the smaller-diameter intracranial vessels functions to reduce cerebral ischemia when used in the prophylaxis and treatment of eclampsia [32].

\section{Efficacy of magnesium sulfate in eclampsia}

$\mathrm{MgSO}_{4}$ has been proven to be an effective first-line treatment for the prevention and treatment of eclampsia. Nonetheless, studies are still being conducted to gauge the degree of effectiveness in comparison to other anticonvulsant drugs. The Cochrane Library was searched for trials that prove the effectiveness of $\mathrm{MgSO}_{4}$. The results show that compared to the placebo/no anticonvulsant group, the mortality rate was reduced by $46 \%$, the risk of eclampsia was reduced by half, and there was decreased risk of placental abruption. The risk of eclampsia was also found to be decreased in patients receiving $\mathrm{MgSO}_{4}$ in comparison to anticonvulsant therapy [4].

Lucas et al. compared the efficacy between $\mathrm{MgSO}_{4}$ and phenytoin. Results showed that 10 out of 1,089 women who were placed in the phenytoin group had eclamptic convulsions, whereas zero of 1,049 women placed in the $\mathrm{MgSO}_{4}$ group had any eclamptic activity. These results corroborate the long-practiced use of $\mathrm{MgSO}_{4}$ in the prevention of eclampsia [5].

A retrospective study conducted in Nigeria by Okereke et al. from 2008 to 2009 focused on the reduction of maternal mortality in eclamptic patients with the use of $\mathrm{MgSO}_{4}$. The study compared the effectiveness of $\mathrm{MgSO}_{4}$ with diazepam. Participants included 1,233 eclampsia patients in the diazepam group and 996 eclampsia patients and 47 preeclampsia patients in the $\mathrm{MgSO}_{4}$ group. Results showed a drastic decrease in the eclampsia case-fatality rate from $20.9 \%$ to $2.3 \%$ among patients in the $\mathrm{MgSO}_{4}$ intervention group [33]. $\mathrm{MgSO}_{4}$ has been recognized as the drug of choice for the treatment of eclampsia and preeclampsia by the World Health Organization (WHO) [34]. Systematic reviews and randomized controlled trials have proven the effectiveness of $\mathrm{MgSO}_{4}$ in preventing these conditions [35]. The evidence, therefore, verifies that $\mathrm{MgSO}_{4}$ is effective in decreasing maternal mortality in eclampsia.

\section{Prophylactic usage of magnesium sulfate}

$\mathrm{MgSO}_{4}$ is used for the prevention of eclampsia in high-risk patients. Despite the drug's proven efficacy, there has been an ongoing debate regarding the correct dosage regimens and their feasibility in resource-limited areas [36]. In this section, we will review the studies that have delved into this topic.

Initially, some physicians continued to use antiepileptics to prevent the occurrence of seizures in high-risk patients. However, this use was not backed by evidence. A comparative study was done by Lucas et al. in 1995 which compared phenytoin and $\mathrm{MgSO}_{4}$ for the prevention of eclampsia in hypertensive patients. They divided pregnant hypertensive women into two groups, one group was allocated treatment with phenytoin $(\mathrm{n}=1,089)$ and the other with $\mathrm{MgSO}_{4}(\mathrm{n}=1,089)$. A total of 10 patients from the phenytoin group developed convulsions whereas none of the $\mathrm{MgSO}_{4}$ patients developed any symptoms indicative of eclampsia [5]. This study was followed by the MAGPIE trial which selected pregnant patients with HTN from 33 countries. In total, 5,071 patients were allocated to $\mathrm{MgSO}_{4}$, while 5,070 patients were given placebo treatment. The study proved that the use of $\mathrm{MgSO}_{4}$ reduces the risk of developing eclampsia by $50 \%$ and lowers the maternal mortality rate. Since this landmark trial, the WHO has promoted the use of $\mathrm{MgSO}_{4}$ for seizure prophylaxis [37].

The traditional regimens of $\mathrm{MgSO}_{4}$, such as those described by Pritchard and Zuspan, require the availability of experienced personnel for administering the medication and monitoring drug toxicity. In low-income areas with a scarcity of resources, the attainability of such ideal conditions can be difficult [38]. This has led to physicians studying and comparing low-dosage regimens with the established regimens for the treatment of eclampsia. 
The Dhaka regimen utilizes a $10 \mathrm{mg}$ loading dose of $\mathrm{MgSO}_{4}$, followed by $2.5 \mathrm{mg}$ given four hours. This is approximately half the dose of the Pritchard regimen and has been found to be equally effective in reducing recurrent seizures [39]. Many studies have now shown that low-dose $\mathrm{MgSO}_{4}$ regimens such as Dhaka regimen, Sokoto regimen, low-dose loading, and maintenance regimens can also adequately manage eclampsia [38]. Such regimens reduce the strain on healthcare resources while maintaining treatment quality and a low incidence of drug toxicity events.

Most of these comparative studies have been carried out in developing countries of Asia. Begum et al. compared a lower-dosage regimen with the higher-dosage regimens and showed that there was equal efficacy in treating eclampsia with both regimens [40]. More recently, a randomized control trial conducted in Nepal compared a single loading dose of $\mathrm{MgSO}_{4}$ with the standard regimen. The trial demonstrated that a single dose is as effective as standard treatment in preventing eclampsia and can be alternatively used for prophylaxis. The results of this trial backed similar findings of Shoaib et al. (Pakistan), Rangana et al. (India), and Murthy et al. (India) reported previously [36,41].

With the above findings in mind, the PIPES trial in 2018 went a step further to compare the single-dose regimen with the low-dose Dhaka regimen in a randomized control trial. Although results did not yield statistically significant differences between the two groups, it was deduced that the shorter regimen of $\mathrm{MgSO}_{4}$ is a viable replacement for the low-dose Dhaka regimen [38]. The aforementioned data demonstrate the efficacy of single, low-dose $\mathrm{MgSO}_{4}$ for the prevention of eclampsia. Larger polycentric trials may be needed to compare it with the 24-hour standard regimen to gain more clarity in updating treatment guidelines.

\section{Cost-effectiveness}

The WHO has acknowledged $\mathrm{MgSO}_{4}$ as the safest, most efficient, and cost-effective medication for the treatment of preeclampsia and eclampsia and has placed it on the Essential Medicines List (EML) for this specific use. Although it is the standard treatment in developed countries, other medications such as diazepam and phenytoin are still widely used in underdeveloped countries. $\mathrm{MgSO}_{4}$ approximately costs $\$ 0.10 / \mathrm{mL}$. From 1995 to 2002, sizable clinical trials found $\mathrm{MgSO}_{4}$ to be most effective in the treatment of preeclampsia and eclampsia compared to other treatment modalities. These trials exhibited more than a 50\% reduction in the incidence of eclampsia and a $46 \%$ reduction in maternal mortality [42].

While $\mathrm{MgSO}_{4}$ is the drug of choice in the treatment of eclampsia, cost remains an issue in developing countries. Routine checking of serum magnesium levels can also become costly. Simon et al. of the MAGPIE trial stated that $\mathrm{MgSO}_{4}$ is most cost-effective when it is limited to patients with severe preeclampsia, which is the precursor to eclampsia. This trial stated that to prevent one case of eclampsia, the cost in USD for 2001 was $\$ 21,202$ in high, $\$ 2,473$ in middle, and \$456 in low-income countries. The cost-effectiveness of $\mathrm{MgSO}_{4}$ would be improved if used only in cases with severe preeclampsia [4].

Another study based on the MAGPIE trial collected data from 9,996 pregnancies: 1,195 from high gross national income (GNI), 5,571 from middle GNI, and 3,230 from low GNI countries. This study assessed the cost of treatment for eclampsia in patients from countries categorized into three distinct groups based on their national income. The results indicated that the number needed to treat in order to prevent one case of eclampsia is 324 in high GNI, 184 in middle GNI, and 43 in low GNI countries. The general cost to counteract one case of eclampsia was $\$ 21,202$ in high, $\$ 2,473$ in middle, and \$456 in low GNI countries. If it is decided to focus $\mathrm{MgSO}_{4}$ treatment on severe preeclampsia, these costs would be lowered to about $\$ 12,942, \$ 1,179$, and $\$ 263$, respectively. If $\mathrm{MgSO}_{4}$ prophylaxis was administered to women with severe preeclampsia, the cost per case would decrease approximately by half. The average cost of $\mathrm{MgSO}_{4}$ treatment which includes the drug and administration is anticipated as $\$ 86$ for high GNI, $\$ 17$ for middle GNI, and $\$ 13$ per person for low GNI countries. It is known that the majority of the costs associated with $\mathrm{MgSO}_{4}$ treatment include drug administration instead of the drug itself, that is, $77 \%, 82 \%$, and $62 \%$, respectively, in high GNI, middle GNI, and low GNI countries [43].

Because drug administration carries most of the weight in terms of cost, many studies have focused on finding alternative methods of drug delivery. If infusion pumps are not readily available for the administration of $\mathrm{MgSO}_{4}$, physicians would have to revert to IV delivery methods, or painful, large-volume IM injections. Another method is a syringe pump known as AutoSyp. This invention was designed to be lowcost, accurate, and low-powered to bypass the cost restrictions in countries with low GNI [44]. Therefore, it has been established that the drug MgSO4 itself is cost-effective, but the means of administering it is expensive. $\mathrm{MgSO}_{4}$ can be made more cost-effective if given prophylactically to preeclamptic patients and if a more affordable method of administration can be recognized.

\section{Anticonvulsants in the treatment of eclampsia}


$\mathrm{MgSO}_{4}$ is the drug of choice to treat patients with eclampsia. It has been proven to be a better agent than other anticonvulsant medications such as benzodiazepines and phenytoin. While it was initially believed that eclamptic seizures have similar pathophysiology as other convulsive diseases, the usual antiseizure medications have not shown to be as beneficial as $\mathrm{MgSO}_{4}$ in controlling eclamptic seizures suggesting otherwise $[4,5,45]$.

$\mathrm{MgSO}_{4}$ initially came to the scene in 1906 after a successful trial was published. It gained prominence in the United States and the United Kingdom during the 1920s, but since the arrival of diazepam and later phenytoin, there persisted a hesitance in the use of $\mathrm{MgSO}_{4}$ for treating eclamptic seizures. This reluctance stemmed from the widespread use of these medications for other forms of seizures, their cost-effectiveness, and availability [45]. The controversy largely ended after a landmark randomized trial was conducted in 1995 (Collab trial) which demonstrated $\mathrm{MgSO}_{4}$ to be superior to diazepam and phenytoin [45]. Trials that followed solidified this evidence. In this section, we will be discussing the results of these trials.

Magnesium Sulfate Versus Diazepam

Diazepam is a cost-effective and readily available agent, especially in rural obstetric centers. It can control convulsions during eclampsia and can treat $\mathrm{HTN}$ and tachycardia [46]. Compared to $\mathrm{MgSO}_{4}$, diazepam does not sufficiently reduce recurrent seizures during an eclamptic episode. A 52\% risk reduction (95\% confidence interval $[\mathrm{CI}]=64-37 \%$ reduction) was reported with the use of $\mathrm{MgSO}_{4}$ [45]. Furthermore, patients treated with diazepam usually required additional therapy to adequately control seizures. $\mathrm{MgSO}_{4}$ has also been shown to have a significantly lower mortality rate compared with diazepam (relative risk [RR] $=0.59$; $95 \%$ CI $=0.37-0.94$ ) [47]. Diazepam has been associated with depressive effects on infants. Infants of mothers who had been treated with diazepam had APGAR scores less than seven at one minute and longer stay in neonatal care units. These low scores persist at five minutes as well $[45,47]$.

Magnesium Sulfate Versus Phenytoin

$\mathrm{MgSO}_{4}$ has proven to be considerably superior to phenytoin in terms of reducing the incidence of recurrent seizures $(\mathrm{RR}=0.31 ; 95 \% \mathrm{CI}=0.20-0.47)$. In addition, those treated with phenytoin have been seen to require auxiliary medications to aid the control of convulsions [45,47]. Improved maternal morbidity outcomes have been observed with the use of $\mathrm{MgSO}_{4}$. Those designated to $\mathrm{MgSO}_{4}$ had a significant reduction in their risk of developing pneumonia $(\mathrm{RR}=0.44 ; 95 \% \mathrm{CI}=0.24-0.79)$, requiring intubation $(\mathrm{RR}=0.66 ; 95 \% \mathrm{CI}=0.49-0.90)$, and intensive care unit admission $(\mathrm{RR}=0.67,95 \% \mathrm{CI}=0.50-0.89)$. Although no significant changes in maternal mortality are observed when comparing the two medications $[4,45,47]$. Phenytoin has substantial effects on infant morbidity as well. Fewer infants of mothers treated with $\mathrm{MgSO}_{4}$ had $\mathrm{APGAR}$ scores less than seven at one minute, required intubation at the time of birth, or required admission in neonatal special care nursery $[45,47]$.

\section{Data table}

Table 1 lists peer-reviewed articles published in English and relevant to the topic. A thorough search on databases such as PubMed, PubMed Central, and Google Scholar was conducted. The following inclusion criteria were used and then decided upon based on the authors' discretion: women without eclampsia; women with preeclampsia; primary objectives including the development of eclampsia, other lifethreatening complications, or death; treatment group receiving $\mathrm{MgSO}_{4}$ as per the local regimen; and a sample size of $>100$. 
toxicity, it is recommended to give $\mathrm{MgSO}_{4}$ prophylaxis to all women with mild preeclampsia [51]. $\mathrm{MgSO}_{4}$ reduces the incidence of eclampsia and lowers maternal mortality, and therefore should be considered for women with preeclampsia who are at risk of developing eclampsia. Its low cost, easy availability, and relatively simple procedure for medication administration and monitoring are advantages. This procedure can be administered by medical personnel, nursing, or even midwifery staff if properly trained [37].

\section{Conclusions}

Eclampsia is a very serious complication of preeclampsia that presents after 20 weeks of gestation. It is associated with increased seizure activity, HTN, and end-organ damage. The CDC has reported a casefatality rate of 6.4 per 10,000 cases of preeclampsia and eclampsia at delivery. The drug of choice to conquer these effects is $\mathrm{MgSO}_{4}$ which exerts its effects on the CNS through the blockade of the NMDA receptors. The Cochrane Library analyzed many trials that proved the efficacy of $\mathrm{MgSO}_{4}$ in the treatment of maternal eclampsia. They proved that the mortality rate decreased by $46 \%$ and the risk of eclampsia was reduced by half in patients receiving treatment with $\mathrm{MgSO}_{4}$. Many other studies compared the effectiveness of $\mathrm{MgSO}_{4}$ with other anticonvulsant medications and the results consistently show $\mathrm{MgSO}_{4}$ as being the most efficacious agent. Prophylactic usage has also been widely recommended by many studies, such as the MAGPIE trial, which suggests that if $\mathrm{MgSO}_{4}$ is given to all mildly preeclamptic patients, the risk of progressing to severe preeclampsia and eclampsia will decrease substantially.

Unfortunately, the use of $\mathrm{MgSO}_{4}$ is constrained in developing countries by the high costs associated with the infusion pump required to administer the drug as well as the intensive monitoring for signs of toxicity. Given that preeclampsia/eclampsia remains one of the most common causes of maternal mortality in low- and middle-income countries, solutions have been proposed to improve the cost-effectiveness of $\mathrm{MgSO}_{4}$, such as limiting its use to severe preeclampsia/eclampsia along with using the IM route and employing alternative low-cost methods of drug delivery. Ultimately, it is well established that $\mathrm{MgSO}_{4}$ is an excellent drug in reducing maternal mortality in eclampsia, concluding that $\mathrm{MgSO}_{4}$ is an effective treatment for the prevention and treatment of eclampsia. With ongoing debate regarding the correct dosage regimens and their feasibility in resource-limited areas, further investigation is recommended for the use of low-dose prophylactic use of $\mathrm{MgSO}_{4}$ in eclampsia.

\section{Additional Information \\ Disclosures}

Conflicts of interest: In compliance with the ICMJE uniform disclosure form, all authors declare the following: Payment/services info: All authors have declared that no financial support was received from any organization for the submitted work. Financial relationships: All authors have declared that they have no financial relationships at present or within the previous three years with any organizations that might have an interest in the submitted work. Other relationships: All authors have declared that there are no other relationships or activities that could appear to have influenced the submitted work.

\section{References}

1. Magley M, Hinson MR: Eclampsia. StatPearls Publishing, Treasure Island, FL; 2021.

2. Vigil-De Gracia P: Maternal deaths due to eclampsia and HELLP syndrome. Int J Gynaecol Obstet. 2009, 104:90-4. 10.1016/j.ijgo.2008.09.014

3. MacKay AP, Berg CJ, Atrash HK: Pregnancy-related mortality from preeclampsia and eclampsia . Obstet Gynecol. 2001, 97:533-8. 10.1016/s0029-7844(00)01223-0

4. Duley L, Gülmezoglu AM, Henderson-Smart DJ, Chou D: Magnesium sulphate and other anticonvulsants for women with pre-eclampsia. Cochrane Database Syst Rev. 2010, 2010:CD000025. 10.1002/14651858.CD000025.pub2

5. Lucas MJ, Leveno KJ, Cunningham FG: A comparison of magnesium sulfate with phenytoin for the prevention of eclampsia. N Engl J Med. 1995, 333:201-5. 10.1056/NEJM199507273330401

6. Luger RK, Kight BP: Hypertension in pregnancy. StatPearls Publishing, Treasure Island, FL; 2021.

7. Roberts JM, Escudero C: The placenta in preeclampsia . Pregnancy Hypertens. 2012, 2:72-83. 10.1016/j.preghy.2012.01.001

8. Kumar V, Abbas A, Aster J: Robbins \& Cotran pathologic basis of disease . Elsevier, Philadelphia, PA; 2021.

9. Roberts JM, Hubel CA: The two stage model of preeclampsia: variations on the theme . Placenta. 2009, 30 Suppl A:S32-7. 10.1016/j.placenta.2008.11.009

10. Warrington JP, George EM, Palei AC, Spradley FT, Granger JP: Recent advances in the understanding of the pathophysiology of preeclampsia. Hypertension. 2013, 62:666-73. 10.1161/HYPERTENSIONAHA.113.00588

11. Tomimatsu T, Mimura K, Endo M, Kumasawa K, Kimura T: Pathophysiology of preeclampsia: an angiogenic imbalance and long-lasting systemic vascular dysfunction. Hypertens Res. 2017, 40:305-10. 10.1038/hr.2016.152

12. Donaldson JO: Invited review: the brain in eclampsia . Hypertens Preg. 1994, 13:115-33. $10.3109 / 10641959409009565$

13. Milliez J, Dahoun A, Boudraa M: Computed tomography of the brain in eclampsia . Obstet Gynecol. 1990, 75:975-80. 
14. Trommer BL, Homer D, Mikhael MA: Cerebral vasospasm and eclampsia. Stroke. 1988, 19:326-9. 10.1161/01.str.19.3.326

15. Belfort MA, Saade GR: Retinal vasospasm associated with visual disturbance in preeclampsia: color flow Doppler findings. Am J Obstet Gynecol. 1993, 169:523-5. 10.1016/0002-9378(93)90612-m

16. Cipolla MJ: Cerebrovascular function in pregnancy and eclampsia . Hypertension. 2007, 50:14-24. 10.1161/HYPERTENSIONAHA.106.079442

17. Dinsdale HB: Hypertensive encephalopathy. Neurol Clin. 1983, 1:3-16. 10.1016/S0733-8619(18)31167-8

18. Tukur J: The use of magnesium sulphate for the treatment of severe pre-eclampsia and eclampsia . Ann Afr Med. 2009, 8:76-80. 10.4103/1596-3519.56232

19. Pritchard JA, Cunningham FG, Pritchard SA: The Parkland Memorial Hospital protocol for treatment of eclampsia: evaluation of 245 cases. Am J Obstet Gynecol. 1984, 148:951-63. 10.1016/0002-9378(84)90538-6

20. Zuspan FP: Problems encountered in the treatment of pregnancy-induced hypertension. A point of view . Am J Obstet Gynecol. 1978, 131:591-7. 10.1016/0002-9378(78)90816-5

21. Okusanya BO, Oladapo OT, Long Q, et al.: Clinical pharmacokinetic properties of magnesium sulphate in women with pre-eclampsia and eclampsia. BJOG. 2016, 123:356-66. 10.1111/1471-0528.13753

22. Chesley LC, Tepper I: Some effects of magnesium loading upon renal excretion of magnesium and certain other electrolytes. J Clin Invest. 1958, 37:1362-72. 10.1172/JCI103726

23. Flowers CE Jr: Magnesium sulphate in obstetrics. A study of magnesium in plasma, urine, and muscle . Am J Obstet Gynecol. 1965, 91:763-76. 10.1016/0002-9378(65)90451-5

24. Hall DG: Serum magnesium in pregnancy. Obstet Gynecol. 1957, 9:158-62.

25. Brandt JL, Glaser W, Jones A: Soft tissue distribution and plasma disappearance of intravenously administered isotopic magnesium with observations on uptake in bone. Metabolism. 1958, 7:355-63.

26. Lu JF, Nightingale $\mathrm{CH}$ : Magnesium sulfate in eclampsia and pre-eclampsia: pharmacokinetic principles . Clin Pharmacokinet. 2000, 38:305-14. 10.2165/00003088-200038040-00002

27. McCubbin JM, Sibai BM, Ardella TN, Anderson GD: Cardiopulmonary arrest due to acute maternal hypermagnesaemia. Lancet. 1981, 1:1058. 10.1016/s0140-6736(81)92225-X

28. MgSO4 dosing and monitoring checklist. (2017). Accessed: August 2, 2021: https://www.unhcr.org/5e0f626c4.pdf.

29. Cotton DB, Janusz CA, Berman RF: Anticonvulsant effects of magnesium sulfate on hippocampal seizures: therapeutic implications in preeclampsia-eclampsia. Am J Obstet Gynecol. 1992, 166:1127-34; discussion 1134-6. 10.1016/s0002-9378(11)90599-7

30. Altura BM, Altura BT, Carella A, Gebrewold A, Murakawa T, Nishio A: Mg2+-Ca2+ interaction in contractility of vascular smooth muscle: Mg2+ versus organic calcium channel blockers on myogenic tone and agonist-induced responsiveness of blood vessels. Can J Physiol Pharmacol. 1987, 65:729-45. 10.1139/y87-120

31. Zheng XY, Yao J, Zhu JM, Li M, Qiu SQ, Zhu ZX, Zhang M: [Effect of magnesium sulfate, nifedipine tablet combined salvia injection on ET-1/NO, TXA2/PGI2 and hemorheology of preeclampsia patients]. Zhongguo Zhong Xi Yi Jie He Za Zhi. 2015, 35:962-5.

32. Belfort MA, Moise KJ Jr: Effect of magnesium sulfate on maternal brain blood flow in preeclampsia: a randomized, placebo-controlled study. Am J Obstet Gynecol. 1992, 167:661-6. 10.1016/s00029378(11)91567-1

33. Okereke E, Ahonsi B, Tukur J, Ishaku SM, Oginni AB: Benefits of using magnesium sulphate $\left(\mathrm{MgSO}_{4}\right)$ for eclampsia management and maternal mortality reduction: lessons from Kano State in Northern Nigeria. BMC Res Notes. 2012, 5:421. 10.1186/1756-0500-5-421

34. Goldenberg RL, Jones B, Griffin JB, Rouse DJ, Kamath-Rayne BD, Trivedi N, McClure EM: Reducing maternal mortality from preeclampsia and eclampsia in low-resource countries--what should work?. Acta Obstet Gynecol Scand. 2015, 94:148-55. 10.1111/aogs.12533

35. Azria E, Tsatsaris V, Goffinet F, Kayem G, Mignon A, Cabrol D: [Magnesium sulfate in obstetrics: current data]. J Gynecol Obstet Biol Reprod (Paris). 2004, 33:510-7. 10.1016/s0368-2315(04)96564-7

36. Rimal SP, Rijal P, Bhatt R, Thapa K: Loading dose only versus standard dose magnesium sulfate seizure prophylaxis in severe pre-eclamptic women. JNMA J Nepal Med Assoc. 2017, 56:388-94. 10.31729/jnma.3431

37. Altman D, Carroli G, Duley L, Farrell B, Moodley J, Neilson J, Smith D: Do women with pre-eclampsia, and their babies, benefit from magnesium sulphate? The Magpie Trial: a randomised placebo-controlled trial. Lancet. 2002, 359:1877-90. 10.1016/s0140-6736(02)08778-0

38. Begum R, Begum A, Johanson R, Ali MN, Akhter S: A low dose ("Dhaka") magnesium sulphate regime for eclampsia. Acta Obstet Gynecol Scand. 2001, 80:998-1002. 10.1034/j.1600-0412.2001.801105.x

39. Keepanasseril A, Maurya DK, Manikandan K, Suriya JY, Habeebullah S, Raghavan SS: Prophylactic magnesium sulphate in prevention of eclampsia in women with severe preeclampsia: randomised controlled trial (PIPES trial). J Obstet Gynaecol. 2018, 38:305-9. 10.1080/01443615.2017.1351931

40. Begum MR, Begum A, Quadir E: Loading dose versus standard regime of magnesium sulfate in the management of eclampsia: a randomized trial. J Obstet Gynaecol Res. 2002, 28:154-9. 10.1046/j.13418076.2002.00029.x

41. Shoaib T, Khan S, Javed I, Bhutta SZ: Loading dose of magnesium sulphate versus standard regime for prophylaxis of pre-eclampsia. J Coll Physicians Surg Pak. 2009, 19:30-3.

42. About magnesium sulfate. (2021). Accessed: June 24, 2021: https://sbccimplementationkits.org/demandrmnch/about-mgso4/.

43. Simon J, Gray A, Duley L: Cost-effectiveness of prophylactic magnesium sulphate for 9996 women with preeclampsia from 33 countries: economic evaluation of the Magpie Trial. BJOG. 2006, 113:144-51. 10.1111/j.1471-0528.2005.00785.x

44. Skerrett E, Kommwa E, Maynard K, Juarez A, Mataya R, Richards-Kortum R, Oden ZM: Evaluation of a lowcost, low-power syringe pump to deliver magnesium sulfate intravenously to pre-eclamptic women in a Malawian referral hospital. BMC Pregnancy Childbirth. 2017, 17:191. 10.1186/s12884-017-1382-9

45. Which anticonvulsant for women with eclampsia? Evidence from the Collaborative Eclampsia Trial . Lancet. 1995, 345:1455-63. 10.1016/s0140-6736(95)91034-4 


\section{Cureus}

46. Dawn CS, Sinha B: Diazepam therapy in eclampsia . Int J Gynaecol Obstet. 1979, 17:281-3. 10.1002/j.18793479.1979.tb00168.x

47. Duley L, Henderson-Smart D: Magnesium sulphate versus phenytoin for eclampsia. Cochrane Database Syst Rev. 2003, CD000128. 10.1002/14651858.CD000128

48. Belfort MA, Anthony J, Saade GR, Allen JC Jr: A comparison of magnesium sulfate and nimodipine for the prevention of eclampsia. N Engl J Med. 2003, 348:304-11. 10.1056/NEJMoa021180

49. Coetzee EJ, Dommisse J, Anthony J: A randomised controlled trial of intravenous magnesium sulphate versus placebo in the management of women with severe pre-eclampsia. Br J Obstet Gynaecol. 1998, 105:300-3. 10.1111/j.1471-0528.1998.tb10090.x

50. Livingston JC, Livingston LW, Ramsey R, Mabie BC, Sibai BM: Magnesium sulfate in women with mild preeclampsia: a randomized controlled trial. Obstet Gynecol. 2003, 101:217-20. 10.1016/s00297844(02)03053-3

51. Berhan Y, Berhan A: Should magnesium sulfate be administered to women with mild pre-eclampsia? A systematic review of published reports on eclampsia. J Obstet Gynaecol Res. 2015, 41:831-42. 10.1111/jog.12697 